\title{
Pneumococcal septic arthritis after splenectomy in Felty's syndrome
}

\author{
Maciej Brzeski, Leslie Smart, Dugald Baird, Robert Jackson, Roger Sturrock
}

\begin{abstract}
A patient with Felty's syndrome who developed bilateral knee septic arthritis and septicaemia due to Streptococcus pneumoniae is described. She had had a previous splenectomy for symptomatic thrombocytopenia, having received pneumococcal vaccine before the operation. Measurement of antibody to the 23 vaccine serotypes showed protective concentrations before infection to just two. The infecting serotype was not represented in the vaccine, but a vigorous antibody response to this serotype occurred. The patient also developed glomerulonephritis due to immune complex deposition.
\end{abstract}

Felty's syndrome is the triad of rheumatoid arthritis and splenomegaly with either neutropenia or thrombocytopenia, occurring in $1 \%$ of patients with rheumatoid arthritis. ${ }^{1}$ Although neutropenia and infections are more common, clinical problems secondary to thrombocytopenia are well recognised and occasionally require splenectomy. ${ }^{1}$ Any subject without a functioning spleen is at risk of severe sepsis, particularly pneumococcal, ${ }^{2}$ and elective splenectomy should be preceded by pneumococcal vaccination. The value of this in Felty's syndrome is unknown. We describe a case of pneumococcal septic arthritis in Felty's syndrome after splenectomy, a complication which, as far as we know, has not been previously reported:

Centre for Rheumatic Diseases, University Department of Medicine, Royal Infirmary, Glasgow

M Brzeski

R Sturrock

Department of Microbiology, Royal Infirmary, Glasgow

D Baird

Department of

Pathology, Royal

Infirmary, Glasgow

R Jackson

Department of

Microbiology,

Stobhill General

Hospital, Glasgow,

Scotland

L Smart

Correspondence to: Dr Maciej Brzes: Rheumatic Diseases Research Dheumatic Discases Research Centre, Stoke Mandeville Hospital, Mandeville Road,
Aylesbury, Bucks HP21 8AL.

Aylesbury, Bucks HP21 8AL. 14 September 1990

\section{Case report}

In June 1989 a 32 year old white woman with rheumatoid arthritis and Felty's syndrome was admitted acutely unwell with a four day history of rigors, fever, and painful bilateral knee effusions.

In 1977 , at the age of 20 , she developed mild, erosive, nodular, seropositive rheumatoid arthritis. Rheumatoid factor was 1/512. Antinuclear antibody test was negative. In November 1984 she was first found to have low white cell and platelet counts, and developed recurrent troublesome epistaxis. Her only treatment was hydroxychloroquine $\mathbf{4 0 0} \mathbf{~ m g / d a y}$ for the preceding 8 months. She had $10 \mathrm{~cm}$ splenomegaly, platelets of $25-50 \times 10^{9} / 1$, and a reduced platelet survival time of 5.3 days (normal 8 days) with excess splenic sequestration. White cell count was $1.9-2.6 \times 10^{9} / 1$ with neutrophils of 0.65 $1 \cdot 2 \times 10^{9} / 1$. Bone marrow showed increased megakaryocytes and adequate leucocyte precursors. A diagnosis of Felty's syndrome was made. She was noted to have generalised skin pigmentation and severely impaired production of tears and saliva. She stopped hydroxychloroquine soon afterwards as her joints were good, but her thrombocytopenia and leucopenia continued unaltered. In September 1985 she had splenectomy for persistent epistaxis and bulk symptoms from splenomegaly - at operation the spleen weighed $860 \mathrm{~g}$. Two weeks before the operation she received a 23-valent pneumococcal vaccine. After splenectomy the platelet count rose to $159-335 \times 10^{9} / 1$ and the neutrophil count to $1 \cdot 0-4 \cdot 2 \times 10^{9} / 1$. In October 1987 nodular regenerative hyperplasia was found on liver biopsy performed because of persistently raised transaminases. Cervical lymphadenopathy was also present, and biopsy showed follicular hyperplasia. In December 1988 she developed cutaneous vasculitis and treatment was started with azathioprine $100 \mathrm{mg}$ orally, with a good response. Her white cell count remained normal during this treatment. Since the onset of rheumatoid arthritis her erythrocyte sedimentation rate has never been below $70 \mathrm{~mm} / \mathrm{h}$ (Westergren) and IgG has been 24-30 g/l (normal 6-15 g/l).

On admission her temperature was $39^{\circ} \mathrm{C}$. She had painful, hot swollen knees, from each of which $30 \mathrm{ml}$ of pus was aspirated. Gram positive cocci were seen in both knee aspirates and Streptococcus pneumoniae type 6A was cultured from both samples and from blood cultures. There was no clinical or radiological evidence of sepsis elsewhere, and she had not experienced recent sinusitis, sore throat, catarrh, or chest infection. Urine analysis, known to be normal four weeks before admission, showed haematuria and proteinuria +++ . Mid-stream urine was sterile. The neutrophil count on admission was $11 \cdot 2 \times 10^{9} / 1$. Azathioprine was stopped and she received $12 \mathrm{~g} /$ day intravenous benzylpenicillin, to which the organism was sensitive. Nose and throat swabs did not yield pneumococcus.

She became afebrile after 48 hours and the right knee returned to normal. Synovitis and effusions persisted at the left knee, but recurrent aspirations were bacteriologically sterile. Needle biopsy of the left knee synovium showed a non-specific synovitis, and no immune complexes or pneumococcal antigen were found. Technical problems, however, might have prevented their detection. One week after admission proteinuria was $1.5 \mathrm{~g} / 24 \mathrm{~h}$. Two months later this had increased to $9 \mathrm{~g} / 24 \mathrm{~h}$. Renal biopsy disclosed a diffuse but non-uniform mesangioproliferative glomerulonephritis. Ultrastructural and immunofluorescence studies strongly suggested a post-infectious cause, and this was confirmed by the demonstration of specific pneumococcal type $6 \mathrm{~A}$ capsular antigen within 


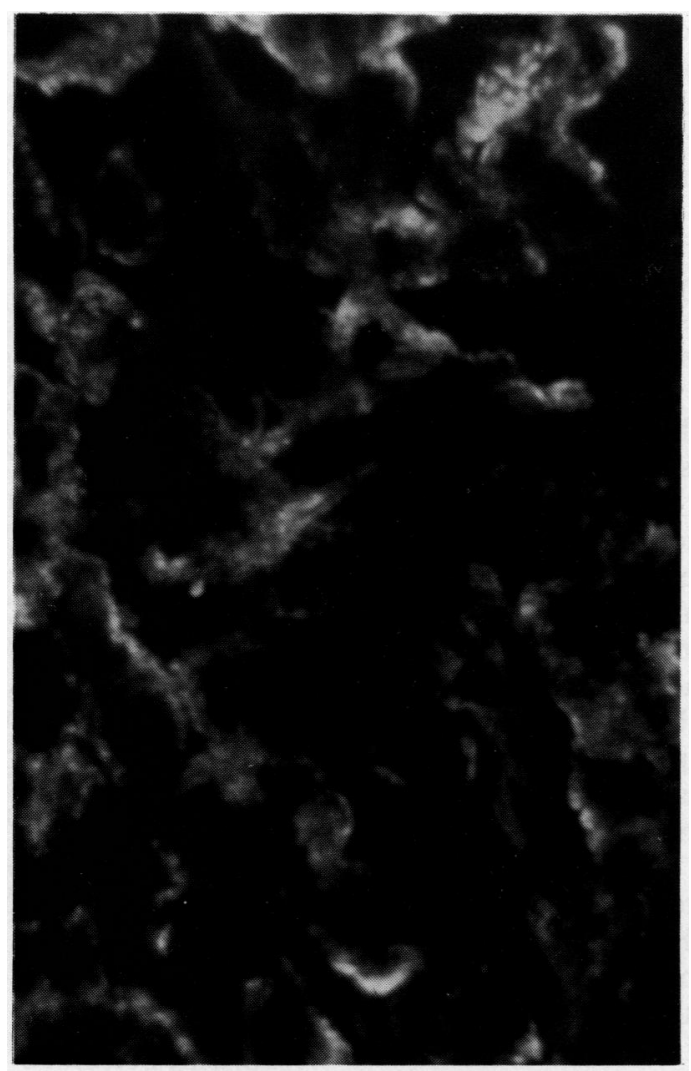

Figure 1 Immune complexes containing the specific pneumococcal antigen are demonstrated within the glomerular mesangium and capillary loops by indirect immunofluorescence (rabbit polyclonal antipneumococcal capsular antigen type 6A).

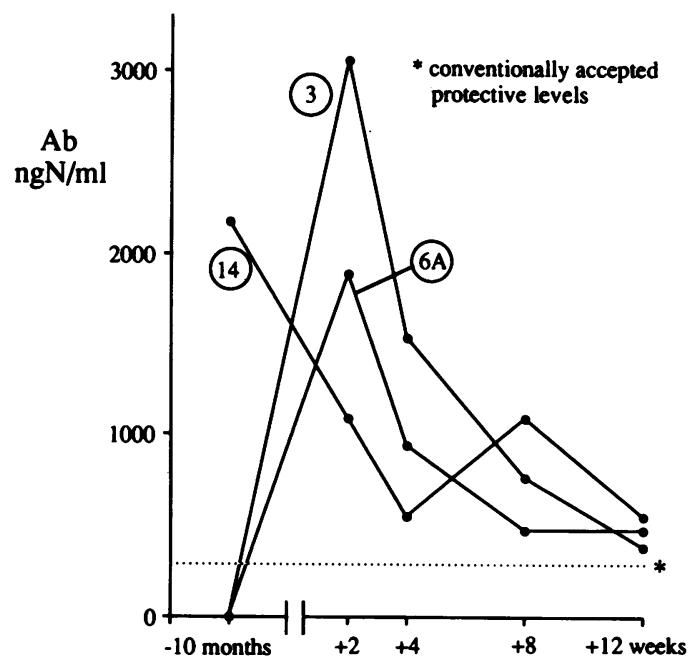

Time from septic arthritis

Figure 2 Antibody $(A b)$ concentrations (in ng immunoglobulin nitrogen/ml) to specific pneumococcal serotypes before and after pneumococcal septic arthritis with type 6A.

the glomerular immune complexes (fig 1). There was no amyloid. Six months after admission proteinuria was $5 \mathrm{~g} / 24 \mathrm{~h}$, she had mild synovitis with effusion of the left knee, and oral penicillin V was stopped.

Antibodies against all 23 pneumococcal vaccine polysaccharides were determined by indirect immunofluorescent assay ${ }^{3}$ carried out on serum taken 10 months before, and two, four, and 12 weeks after this infection. 'Protective'4 concentrations were found for only two types (14 and 15B) four years after vaccination. The patient produced a satisfactory response to type 6A (not represented in the vaccine) and also developed an anamnestic response to types 3 (fig 2) and 11A. Ten weeks after onset of infection total IgG was $27 \cdot 5 \mathrm{~g} / \mathrm{l}$ (normal 6-15 g/l) with IgG2 $1.9 \mathrm{~g} / \mathrm{l}$ (normal 1.0-6.5 g/l).

\section{Discussion}

As far as we know this is the first description of pneumococcal septic arthritis in Felty's syndrome, and the first proved report of pneumococcal septic arthritis after splenectomy for any indication in an adult. The infecting serotype in our patient was not present in the pneumococcal vaccine she had received before splenectomy. Furthermore, she developed a pneumococcal associated glomerulonephritis.

Although patients with Felty's syndrome have a high susceptibility to infections, ${ }^{5}$ this is not due to neutropenia alone, and restoration of normal neutrophil counts by splenectomy does not always reduce infections. ${ }^{6}$ Septic arthritis has been reported infrequently, 78 perhaps because Felty's syndrome is itself uncommon. Septic arthritis after splenectomy is rare in adults. Two possible but unconfirmed cases of pneumococcal septic arthritis after splenectomy have been reported. Thirty three years after splenectomy for idiopathic thrombocytopenia one patient had pneumococcal endocarditis and possible infection of two previously normal metacarpophalangeal joints. ${ }^{9}$ The second patient developed a swollen knee associated with pneumococcal septicaemia two days after splenectomy for haemolytic anaemia. ${ }^{10}$

It is likely that previous splenectomy is an important predisposing factor. There is a high risk of severe pneumococcal sepsis after splenectomy. ${ }^{211}$ Patients who have undergone splenectomy have an impaired response to pneumococcal vaccine, ${ }^{12}$ perhaps due to a persistent B cell defect impairing synthesis of IgM against pneumococcal capsular polysaccharide. ${ }^{13}$ Vaccination should thus be given before the operation whenever possible. In rheumatoid arthritis it is not clear whether. this is worthwhile as there is evidence that patients have a diminished response to immunisation. ${ }^{14}$ Our patient had a relatively low IgG2 concentration, and this is recognised in association with pneumococcal infection, possibly as a predisposing factor. ${ }^{15}$ Treatment with azathioprine might also have predisposed our patient to sepsis. Her absolute neutrophil count was known to be normal at a monitoring visit two weeks before admission, however, and the dose of azathioprine had been unchanged for six months.

The vaccine covers the 23 most commonly isolated pathogenic pneumococcal serotypes, accounting for up to $94 \%$ of isolates in the west of Scotland. ${ }^{16}$ In our case the infecting serotype was $6 \mathrm{~A}$, not present in the vaccine, though 6B (which is) has been claimed to have some cross reactivity and therefore to offer possible protection. ${ }^{4}$ Failure of vaccination to prevent subsequent sepsis has been reported, ${ }^{17}$ and our case is a further example. 
Antibody concentrations of $200-300 \mathrm{ng}$ immunoglobulin nitrogen $/ \mathrm{ml}$ are generally taken as protective against subsequent infection. ${ }^{4}$ The duration of benefit of pneumococcal vaccination is unknown. This is important because overwhelming pneumococcal sepsis may occur up to 40 years after splenectomy. ${ }^{11}$ Measurement of antibody four years after vaccination in our patient suggests that she had protective concentrations against only two of the 23 serotypes in the vaccine. The normal antibody response to the infecting serotype shows that the patient is still able to produce adequate antibody, and suggests that repeat vaccination should be considered despite the studies previously quoted. ${ }^{13} 14$ Data on long term antibody concentrations in vaccinated patients with Felty's syndrome are not available; anecdotal experience suggests that four yearly vaccination might be desirable and worth investigating. Penicillin prophylaxis is contentious, but poor complicance or emergence of resistant strains would undermine any potential benefit.

Pneumococcal associated glomerulonephritis is a rare phenomenon. There is little doubt that the glomerular lesion in this case is directly related to the septic arthritis as the capsular antigen of the specific infecting serotype was detected within the glomerular immune complexes. Moreover, the clinical and renal pathological features of our case, although not typical of the classical poststreptococcal (pyogenes) glomerulonephritis, are remarkably similar to those described in previous reports of glomerulonephritis associated with pneumococcal pneumonia. ${ }^{18}{ }^{19}$ The persistence of severe proteinuria despite adequate treatment of the acute infection may be related to reduced clearance of circulating immune complexes as a result of the splenectomy. It is interesting to speculate whether the persistent synovitis might also be related to immune complex deposition.

Sepsis after splenectomy can start as a mild illness and accelerate catastrophically. Patients should be made aware of possible early symptoms of sepsis, and their medical attendants should not dismiss as trivial early symptoms of a potentially lethal condition. A 'postsplenectomy card', similar to those carried by patients with prosthetic joints or those receiving prednisolone or warfarin, might help. ${ }^{20}$

1 Pinals R S. Felty's syndrome. In: Kelly W N, Harris E D, Ruddy S, Sledge C B, eds. Textbook of rheumatology. Philadelphia: Saunders, 1989: 993-8.

2 Bisno A L. Hyposplenism and overwhelming pneumococca infection: a reappraisal. Am f Med Sci 1971; 262: 101-7.

3 Sloyer J L, Howie V M, Ploussard J H, Amman A J, Austrian $\mathbf{R}$, Johnston $\mathbf{R} B$. Immune response to acute otitis media in $\mathbf{R}$, Johnston $\mathbf{R}$ B. Immune response to acute otits media in
children. 1 . Serotypes isolated and serum and middle ear children. 1. Serotypes isolated and serum and middle ear 1974; 9: 1028-32.

4 Robbins J B, Austrian R, Lee C-J, et al. Considerations for formulating the' second-generation pneumococcal capsular polysaccharide vaccine with emphasis on the cross reactive types within groups. F Infect Dis 1983; 148: 1136-59.

5 Laszlo J, Jones R, Silberman H R, Banks P M. Splenectomy for Felty's syndrome. Clinicopathological study of 27 patients. Arch Intern Med 1978; 138: 597-602.

6 Thorne C, Urowitz M B. Long term outcome in Felty's syndrome. Ann Rheum Dis 1982; 41 : 486-9.

7 Moore R A, Brunner C M, Sandusky W R, Leavell B S. Felty's syndrome: long-term follow-up after splenectomy. Ann Intern Med 1971; 75: 381-5.

8 Breedveld F C, Fibbe W E, Cats A. Neutropenia and infections in Felty's syndrome. Br $\mathcal{F}$ Rheumatol 1988; 27 : 191-7.

9 Bruyn G A W, Thompson J, van der Meer J W M. Pneumococcal endocarditis in adult patients. A report of five cases and review of the literature. $Q \mathcal{F}$ Med 1990; 74: 33-40.

10 Selby C, Hart S, Ispahani P, Toghill P J. Bacteraemia in adults after splenectomy or splenic irradiation. $Q \mathcal{F ~ M e d}$ 1987; 63: 523-30.

11 Francke E L, Neu H C. Postsplenectomy Infection. Surg Clin North $A m$ 1981; 61: 135-55.

12 Hosea S W, Brown E J, Burch C G, Berg R A, Frank M M Impaired immune response to polyvalent pneumococcal vaccine. Lancet 1981; i: 804-7.

13 Di Padova F, Durig M, Harder F, Di Padova C, Zanussi C. Impaired antipneumococcal antibody production in patients without spleens. BMF 1985; 290: 14-16.

14 Bucknall R, Bacon P, Elson C, Jones J V. Antibody producing capacity to the bacteriophage $0 \times 174$ in rheuma producing capacity to the bacteriophage 0X174.
toid arthritis. Ann Rheum Dis 1987; 46: 889-97.

15 Haeny M. Clinical aspects of antibody deficiency. Hospital Update 1990; 16: 122-34.

16 Smart L E, Dougall A J, Girdwood R W A. Pneumococcal bacteraemia. $B M \mathcal{F}$ 1985; 290: 935

17 Brivet F, Herer B, Fremaux A, Dormont J, Tchernia G Fatal post splenectomy pneumococcal sepsis despite pneumococcal vaccine and penicillin prophylaxis. Lancet 1984 ii: 356-7.

18 Kaehny W D, Ozawa T, Schwarz M I, Stanford R E, Kohler $P$ F, McIntosh $R$ M. Acute nephritis and pulmonary alveolitis following pneumococcal pneumonia. Arch Intern Med 1978; 138: 806-8.

19 Hyman L R, Jenis E H, Hill G S, Zimmerman S W Burkholder $\mathbf{P} M$. Alternate $\mathrm{C} 3$ pathway activation in purkholder $\mathrm{P} M$. Alternate C3 pathway activation in pneum.

20 Duncombe A S, Dudley J M, Slater N G P, Treacher K F. Overwhelming pneumococcal sepsis post-splenectomy. Lancet 1987; i: 570. 